\title{
Design and Fabrication of Microchannels for Magnetohydrodynamic Flow
}

\author{
Jian-Bin Bao and D. Jed Harrison \\ Department of Chemistry, University of Alberta, Edmonton, AB T6G 2G2, Canada \\ E-mail: jbao@ualberta.ca
}

\begin{abstract}
This paper introduces a new microchannel design for magnetohydrodynamic (MHD) flow that maximizes MHD pumping power. An electromagnetic channel (EMC), which has a rectangular cross-section and electrodes that run the full length of the channels, is the key design element. EMC fabrication on Si requires a two-mask process. ICP-RIE was employed to open rectangular grooves, and then an insulation layer and a seed layer for electroplating were sputtered into these grooves. Current densities for electroplating were adjusted to obtain as planar surface as possible. However, a polishing process needed to be developed to achieve the required planarity for subsequent bonding. Profilometry showed that the surface roughness after polishing was less than 200nm, which was adequate for bonding. The conditions for the second ICP-RIE step, which opened a $10 \mu \mathrm{m}$ wide channel between the electrodes, were adjusted to protect the electrodes. Finally, HF bonding was employed to bond the wafer to an O211 glass coverplate. Bubble voltage threshold tests indicated $2.2 \mathrm{~V}$ could be applied within the device at $960 \mathrm{~Hz}$ before electrolysis created gas bubbles. These voltages are high enough to give the required pumping velocities for open tubular liquid chromatography.
\end{abstract}

\section{Introduction}

Electroosmotic flow has been successful to date, in part because on a chip it is a challenge to manipulate traditional head pressure driven flow without active valving. Magnetohydrodynamic (MHD) flow could be very important on-chip because it creates a pressure driven flow and allows flow control without mechanical valves [1, 2]. Unfortunately, the flow velocity is inversely proportional to the square of channel dimensions, so this pump scales in the wrong direction for microfluidics. This report explores designs to resolve drawbacks in using MHD to perform open tubular chromatography in narrow channels, using an electromagnetic channel (EMC) to achieve powerful pumping in narrow channels.

\section{Design}

MHD does not generate high enough pressure for packed bed chromatography, and open tubular chromatography requires narrow channels (10 $\mu \mathrm{m}$ or less), making the design of MHD devices challenging.

Lee [1] stated the pressure generated by an AC MHD is:

$$
p=I B / D
$$

where $p$ is pressure, $I$ is the electric current across a channel, $B$ the magnetic field, and $D$ the height of the electrodes. Current is limited by the bubble current density threshold $j=I / I D$, at which significant electrolysis occurs, so we can also write

$$
p=j l B
$$

where $l$ is the length of the electrodes. Clearly we can enhance the pumping power by extending the electrodes the full length of the channel.

A trapezoidal channel cross-section is easy to make using wet anisotropic Si etching [1], but depositing metal on the walls gives non-parallel electrodes. The nonuniform $j$ this causes in the channels negatively impacts the flow velocity that can be attained. For a given bubble current density threshold we can use the empirical equation of flow resistance for a trapezoidal cross-section [3] to calculate the mean flow velocity, $v$, for MHD flow

$$
v=\frac{j B D W \sin ^{2} \theta}{16 \eta \cot \theta(1+\sin \theta)^{2}} \ln \frac{W+2 D \cot \theta}{W}
$$

where $W$ is the minimum width of the channel, $\eta$ the viscosity of the fluid, and $\theta$ is the trapezoidal angle, smaller than $90^{\circ}$.

Table 1 shows the relative mean velocities generated by channels with different cross-sectional shapes, but the

Table 1. Relative mean velocities (square channel as reference) generated by channels with the same cross-sectional area and depth but different shapes

$\begin{array}{ccc}\text { Cross-section } & \theta & \text { Relative mean velocity } \\ \text { trapezoidal } & 54.7 & 29.4 \\ \text { trapezoidal } & 60 & 41.5 \\ \text { rectangular } & 90 & 100\end{array}$


a) Lithography/ICP-RIE

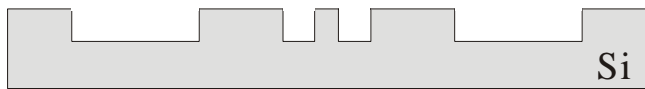

b) Deposit/Lift off

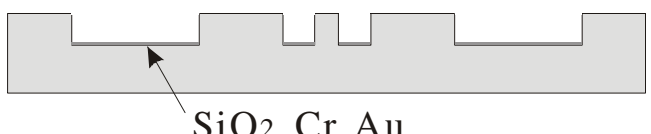

c) Electroplate/Polishing

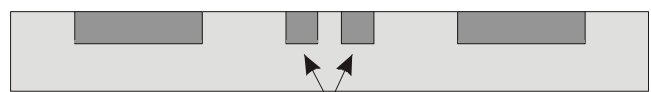

Electrode d) Lithography/ICP-RIE

Channel

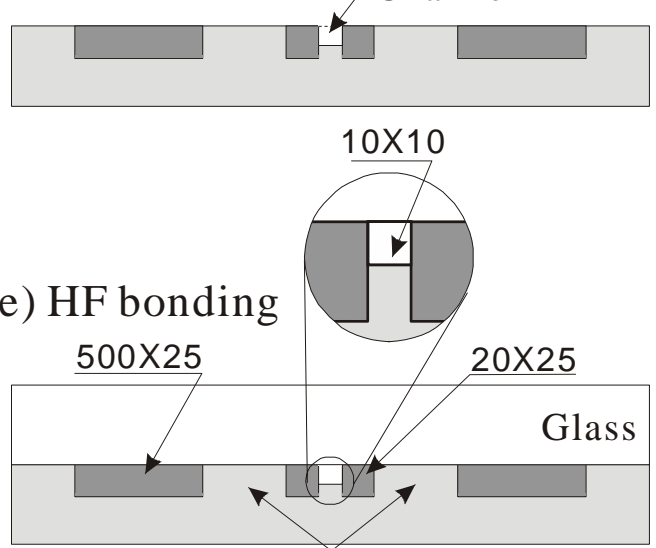

Bonding Nail

Figure 1. Process steps for the fabrication of EMC for MHD flow. The cross-section of the EMC is shown as the inset in e)

same area and depth. The flow velocity will reach a maximum at $\theta=90^{\circ}$. Thus the best design is rectangular, with metal sidewalls. We refer to a rectangular cross section with electrodes that run the full length of the channels as an EMC. By using EMC, the dependence of the pumping velocity on the channel length for a traditional pressure pump is not an issue. We calculate an average velocity of $0.12 \mathrm{~mm} / \mathrm{sec}$ could be achieved at 0.45 $\mathrm{T}$ with this design, for channels with a width and depth of 10 by $10 \mu \mathrm{m}$, a voltage of $2 \mathrm{~V}$ and a frequency of $960 \mathrm{~Hz}$.
The leads contacting the channels need to have a large cross-section to avoid significant potential differences along the channels due to electrode impedance, given that currents of $\sim 100 \mathrm{~mA}$ will be needed. Flexible control of flow within each channel can be obtained using independent contact electrode pairs within each channel segment. This combination of design elements has not been used previously within an MHD chip, but could result in reasonable pumping speeds.

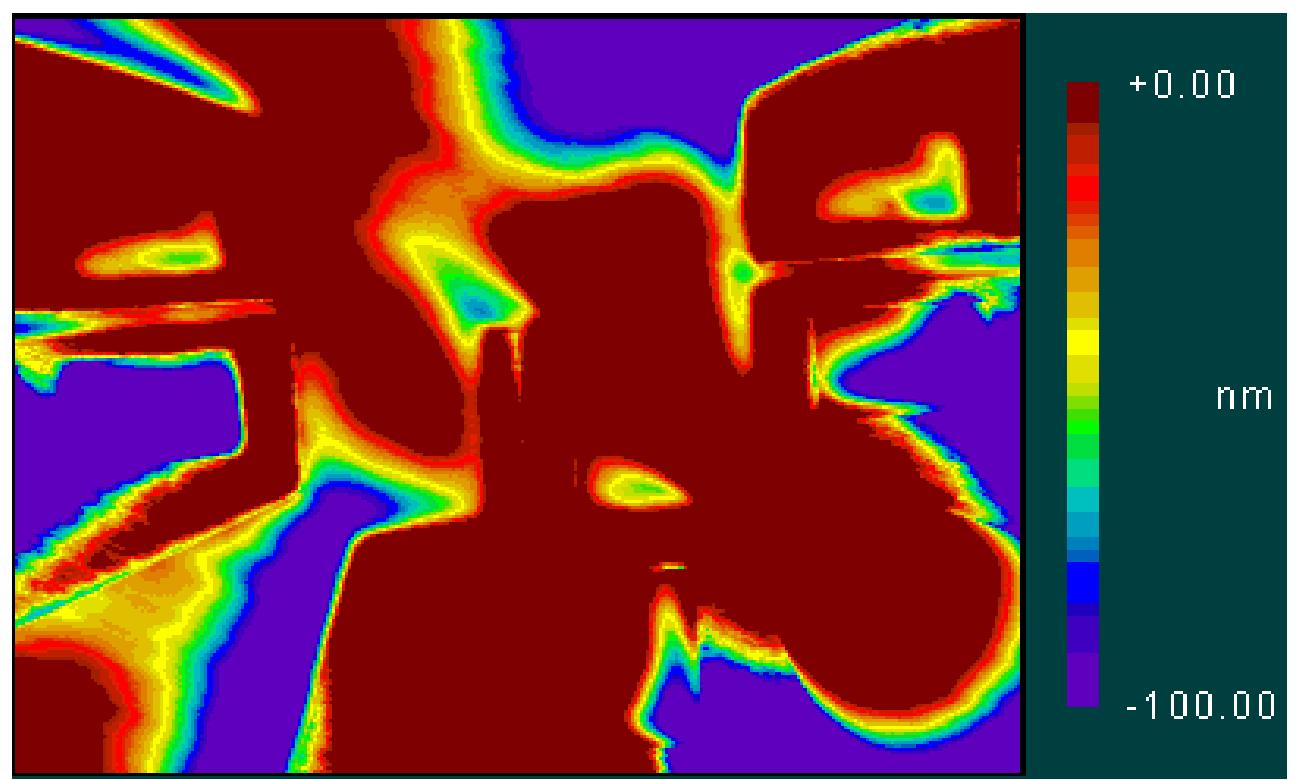

Figure 2. Surface profile in the vicinity of a T-junction from optical profilometer. 


\section{Fabrication}

The EMC design presents a significant challenge for current microfabrication methods, although the issues are not obvious at first glance. The EMC design requires a top plate be bonded to create capillaries by capping the channels formed in the bottom plate, placing severe constraints on the planarity of the bottom plate. Thick metal deposits have usually been made by electrochemical deposition. While this method is common in micromachining, there is no significant experience with placing a hermetically sealed top plate over such electroplated structures. In fact, the roughness of most electroplated surfaces means this can not be accomplished without additional processing. We have developed a polishing procedure to address this issue.

The required feature size of $10 \mu \mathrm{m}$ made SU-8 unacceptable as a deep resist, as $15 \mu \mathrm{m}$ is a typical lower feature size [4], meaning bulk machining with ICP-RIE was preferred to obtain vertical sidewalls. A two-mask process was employed, as indicated in Figure 1. Silicon dioxide was deposited by an e-beam evaporator to prevent electrical contact to the Si substrate, and a patterned seed layer of $\mathrm{Cr} / \mathrm{Au}$ was used to initiate electroplating of $\mathrm{Ni}$, $\mathrm{Au}$, or Ag. It is important that the photoresist (HPR 504 in this case) not be damaged by the plasma while doing ICPRIE. The evaporation rate of silicon dioxide was carefully controlled to be less than $5 \AA / s e c$, to keep the surface temperature low and avoid damaging the HPR504. A routine $\mathrm{Cr} / \mathrm{Au}$ sputter deposition process was employed, because HPR 504 survived the argon plasma.

It is a big challenge to get uniform electroplating surface for a patterned wafer, because of unavoidable, non-uniform lines of current [4] and the resulting metal ion concentration gradients in the electroplating solution. This is true even with patented commercial deposition solutions (Technic Canada, Inc). Slow plating gives improved results. In this work, with current densities less than $3.5 \mathrm{~mA} / \mathrm{cm}^{2}$ for nickel, and less than $1 \mathrm{~mA} / \mathrm{cm}^{2}$ for silver, the thinnest wires (20 $\mu \mathrm{m}$ wide) grew almost twice as fast as the widest wires (500 $\mu$ m wide).

Bonding required less than $0.2 \mu \mathrm{m}$ surface high variation. This was achieved by polishing with $50 \mathrm{~nm}$ $\mathrm{Al}_{2} \mathrm{O}_{3}$ particles prepared as a paste in distilled water. $\mathrm{A}$ polishing cloth was affixed to a planar pad and loaded into a drill. Polishing was done at a speed less than 100 RPM so as not to break the wafer, requiring several hours. Figure 2 shows several typical surfaces after polishing.

A second mask step was then used with ICP-RIE to etch a flow channel between the electrodes. It was suspected that gold or silver would contaminate the ICPRIE. [5] Using the Bosch process, we found it helpful to
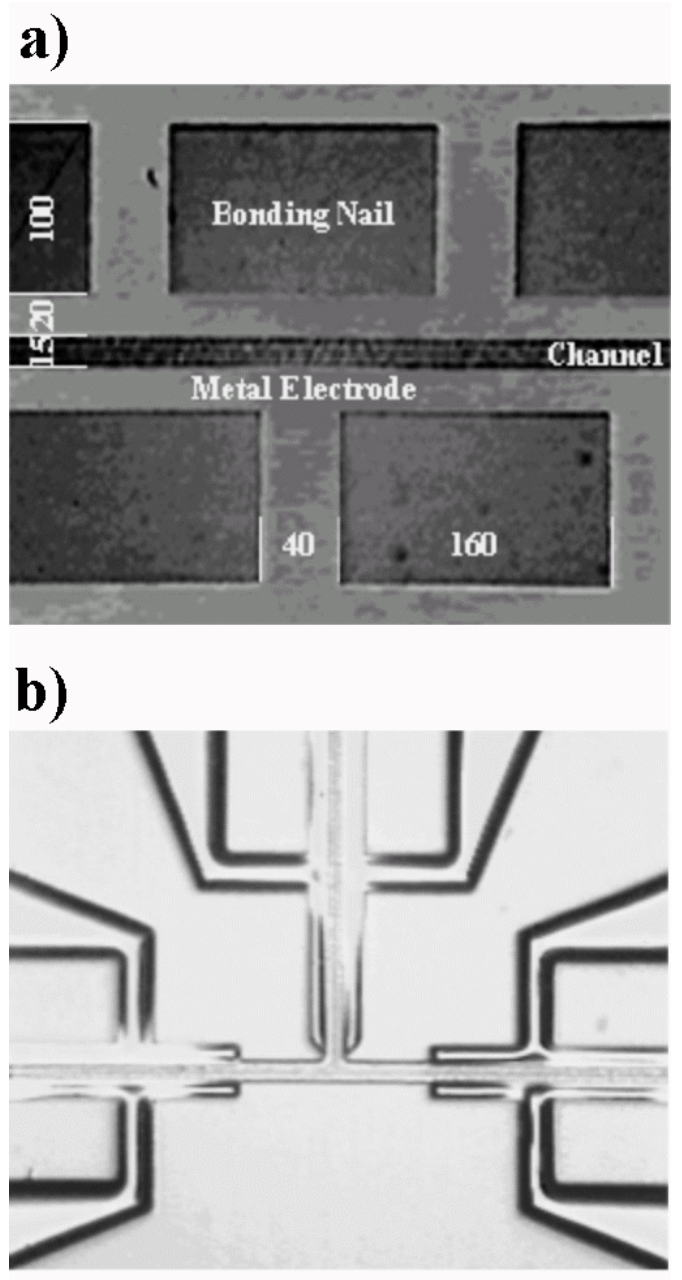

c)

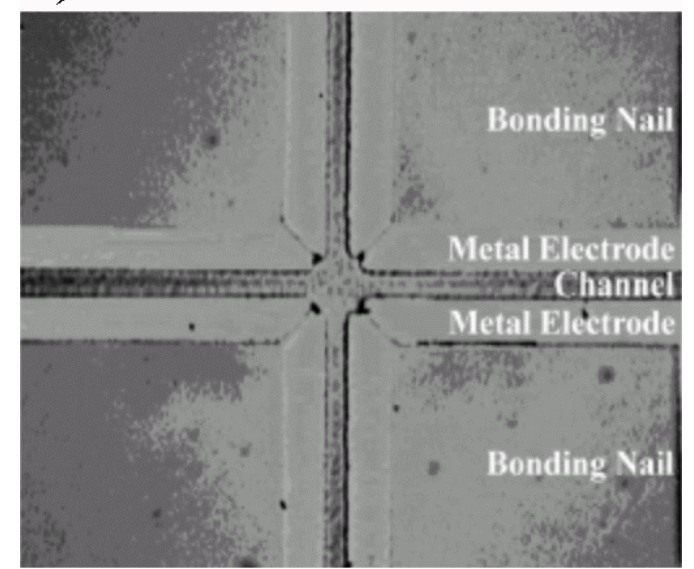

Figure 3. Photomicrographs for MHD channels in different shapes. a) Straight channel; b) T-junction, with three pairs of isolated electrodes; c) Cross junction, with four pairs of electrodes 
halve both etching and deposition times. Doing so apparently lowered the possibility of damaging the electrodes with fluorine or sulphur radicals. This adjustment works especially well given that in this step shallow channels $(10 \mu \mathrm{m})$ were fabricated.

Finally, an $\mathrm{O} 211$ glass cover plate was bonded to the silicon wafer by $\mathrm{HF}$ bonding [6], at $20^{\circ} \mathrm{C}$. Glass does not bond to metal, so to ensure bonding, near the flow channel an arrangement of bonding nails embedded within the metal deposit and polished flush with it was used. These may be seen in Figure 3a.

\section{Characterization and Discussion}

The problems in metallizing and planarizing MHD devices are actually very similar to current metallization challenges in ULSI microchip fabrication. Chemical mechanical polishing (CMP) with a slurry has been widely used in the damascene process to metallize $\mathrm{Cu}$ vias formed in ULSI microchips. Silver may in fact be the material used for the next ULSI generation, but CMP of patterned silver is in its infancy [7]. We have shown a high planarity can be achieved by oxide particle polishing, without any chemical agents. We believe this $\mathrm{Ag}$ patterning technique could be very useful for the IC industry.

Figure 3 shows photos of three typical, realized device elements: a straight channel, T-junction, and cross junction. Electrodes in each channel segment can be individually addressed. Electrode geometry near intersections, illustrated in Figure 3b, was designed to prevent electrical leakage and cross-talk, by separating the electrodes by at least $75 \mu \mathrm{m}$. About $2 \mathrm{~V}$ is required to obtain the currents for running chromatography. Tests on bubble voltage threshold (Figure 4) were done with $\mathrm{Ni}$ electrodes formed in the chip, showing higher than $2 \mathrm{~V}$

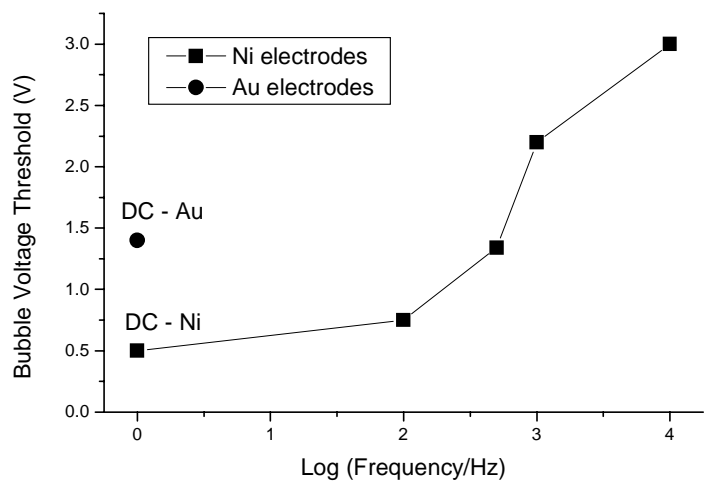

Figure 4. Bubble voltage threshold for $1 \mathrm{M} \mathrm{NaCl}$ solution as a function of frequency could be applied at $1 \mathrm{kHz}$. The higher DC threshold of Ag and Au suggests much higher currents will be possible in devices made with either of these metals. This would allow still higher currents and pumping rates, or the use of lower frequencies and higher magnetic fields.

\section{Conclusion}

We have designed and fabricated an EMC which can generate more powerful MHD pumping in a channel with a $10 \mu \mathrm{m}$ square cross section. The bubble threshold we have achieved will allow flow rates high enough for performing open tubular chromatography. The channel size is small enough for this chromatographic method to be effected. The challenges associated with fabrication have been overcome by optimizing electroplating conditions, developing polishing techniques for planarization of rough electrochemical surfaces, and reducing the ICP-RIE etching rates.

\section{References}

1. A. V. Lemoff, and A. P. Lee, "An AC Magnetohydrodynamic Micropump”, Sensors \& Actuators B: Chemical, 2000, 63, 178-185

2. J. Jang, and S. S. Lee, "Theoretical and Experimental Study of MHD (Magnetohydrodynamic) Micro-pump,” Sensors \& Actuators A: Physical, 2000, 80, 84-89

3. E. Fried, and I. E. Idelchik, Flow Resistance: A Design Guide for Engineers, Hemisphere, New York, 1989

4. J. W. Judy, "Judylab Recipes and Procedures", http://www.ee.ucla.edu/research/judylab/procedures/

5. M. A. Schmidt, Private communication

6. H. Nakanishia, T. Nishimotoa, R. Nakamurab, A. Yotsumotob, T. Yoshidaa, and S. Shojib, "Studies on $\mathrm{SiO}_{2}-\mathrm{SiO}_{2}$ Bonding with Hydrofluoric Acid. Room Temperature and Low Stress Bonding Technique for MEMS”, Sensors \& Actuators A: Physical, 2000, 79, $237-$ 244

7. M. Hauder, J. Gstottner, L. Gao, and D. SchmidtLandsiedel, "Chemical Mechanical Polishing of Silver Damascene Structures”, Microelectronic Engineering, 2002, 64, 73-79 